\title{
Consumer Preferences for Skin Cancer Screening Using Mobile Teledermoscopy: A Qualitative Study
}

\author{
Fleur Kong ${ }^{a}$ Caitlin Horsham ${ }^{a, b}$ Jenna Rayner ${ }^{c}$ Marko Simunovic ${ }^{b}$ \\ Montana O'Hara ${ }^{a, b}$ H. Peter Soyer ${ }^{c}$ Monika Janda ${ }^{a, b}$ \\ ${ }^{a}$ Centre for Health Services Research, Faculty of Medicine, University of Queensland, Brisbane, QLD, Australia; \\ ${ }^{b}$ School of Public Health and Social Work, Institute of Health and Biomedical Innovation, Queensland University \\ of Technology, Brisbane, QLD, Australia; ${ }^{C}$ Dermatology Research Centre, University of Queensland Diamantina \\ Institute, University of Queensland, Brisbane, QLD, Australia
}

\section{Keywords}

Melanoma · Skin cancer · Mobile teledermoscopy · Cancer screening

\begin{abstract}
Background: Mobile teledermoscopy is a rapidly advancing technology that promotes early detection and management of skin cancers. Whilst the use of teledermoscopy has proven to be effective and has a role in the detection of skin cancers, patients' attitudes towards the multiple ways in which this technology can be utilised has not been explored. Methods: Data were obtained from a large randomised controlled trial comparing mobile teledermoscopy-enhanced skin self-examinations (SSEs) with naked-eye SSE. A semi-structured interview guide was developed by the investigators with questions focusing on people's previous skin screening behaviours and 2 of the major pathways which can be utilised in mobile teledermoscopy: (i) direct-to-consumer and (ii) doctor-to-doctor. All interviews were tape-recorded and transcribed verbatim. Thematic analysis was undertaken by 2 independent researchers. Results: Twenty-eight participants
\end{abstract}

were interviewed. Eighty-six percent of participants ( $n=$ $24 / 28$ ) had previously had a clinical skin examination. Only $18 \%$ of participants $(n=5 / 28)$ visited the same doctor for each clinical skin examination. Five main themes were identified in the interviews that affected how people felt about the integration of mobile teledermoscopy into skin screening pathways: history of clinical skin examinations, continuity of the doctor-patient relationship, convenience of the direct-to-consumer teledermoscopy, expedited review enhancing the doctor-to-doctor setting and mobile teledermoscopy as a partner-assisted task. Conclusions: Overall mobile teledermoscopy was viewed positively for both direct-to-consumer and doctor-to-doctor interaction. Continuity of care in the doctor-patient relationship was not found to be a priority for clinical skin examination with most participants visiting several doctors throughout their clinical skin examination history.

(c) 2020 S. Karger AG, Basel

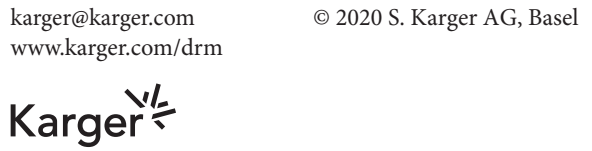

Monika Janda

Centre for Health Services Research, Faculty of Medicine

University of Queensland, Building 33, Princess Alexandra Hospital Campus

Woolloongabba, QLD 4102 (Australia)

m.janda@uq.edu.au 
Fig. 1. Flowchart of Materials and Methods. SSE, skin self-examination.

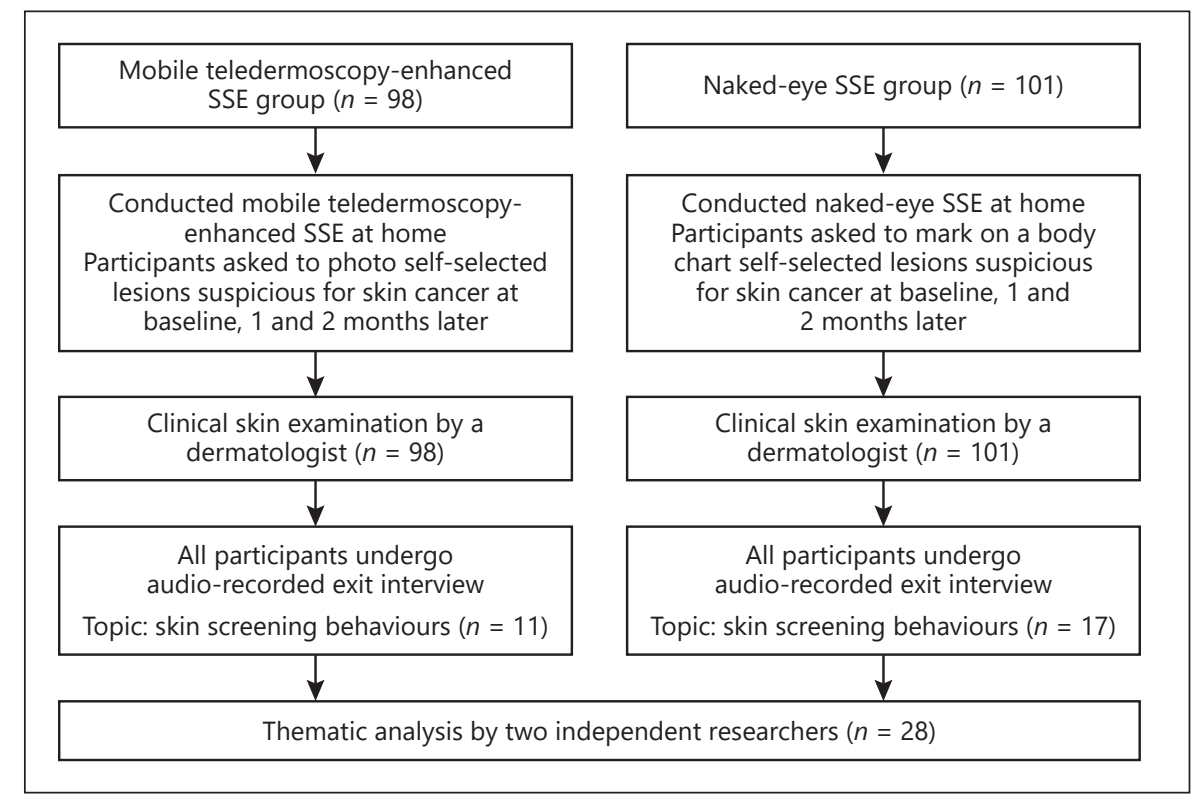

\section{Introduction}

Medical diagnostics and treatments are increasingly offered directly to patients using digital technology or applications (apps) [1]. Mobile teledermoscopy is a rapidly advancing technology that promotes early detection and management of skin cancers such as melanomas [2]. Skin cancers are commonly visible on the skin surface and can therefore be photographed and potentially diagnosed via remote telediagnostic assessment. Mobile teledermoscopy is a store-and-forward technology that uses a smartphone with a dermatoscope attachment in conjunction with an associated app to capture, track and send photos for remote dermatology diagnosis [3]. Mobile teledermoscopy has a wide range of applications, and consequently there has been an exponential increase in available smartphone apps for users to send in photos of skin lesions of concern for diagnosis [4]. Teledermatology has been evaluated for its efficacy, with a recent systematic review of the available evidence concluding that the correct diagnosis of skin lesions as malignant using photographic images had a sensitivity of $94.9 \%$ and a specificity of $84.3 \%$ [5]. These results implicate teledermatology as a valuable tool in the diagnosis of skin cancer.

In this research we refer to 2 clinical care settings in which mobile teledermoscopy may be used [5]. In the direct-to-consumer setting, patients take photos of skin lesions during a skin self-examination (SSE) and send them directly to a doctor. These can be either lesions a doctor has identified and recommended the patient to monitor over time or lesions the patients select themselves as suspicious for skin cancer. In the direct-to-consumer model either (i) the patient knows the doctor/clinic or (ii) there is no prior relationship between the consumer and doctor/clinic. When photographs are submitted via the direct-to-consumer model, research has shown a good diagnostic concordance compared to clinical skin examinations [6], and using this method may therefore allow patients to reduce the number of clinical skin examinations they require. The second setting is the doctor-todoctor setting, where the teledermoscopy process is used to triage urgent cases into specialist dermatological care more quickly. General practitioners (GPs) or other health professionals who examine patients' skin can receive a second opinion by dermatologists or skin cancer specialists, increasing their confidence and scope of practice. A recent systematic review found teledermatology is costeffective and works well as a triaging tool [3].

These virtual clinical care settings for skin cancer diagnosis and surveillance may change the doctor-patient relationship. Dobson [7] described how attitudes towards health care-related smartphone apps vary widely and often differ between health professionals and patients. Therefore, to satisfy both the patient and the doctor, it is necessary to understand the views of both parties for these new virtual clinical care settings [7]. The feasibility and accuracy of mobile teledermoscopy has been studied previously, along with its barriers [8] and doctors' and 
patients' acceptability [2]. However, few studies have specifically assessed whether acceptability differs depending on a patient's regular clinical skin examinations by a doctor and their opinion on the utility of the different settings of teledermoscopy as described above. This study aimed to explore consumer views on skin screening services for skin cancer by interviewing recent users of mobile teledermoscopy as part of their participation in a randomised controlled trial.

\section{Materials and Methods}

For further details, see the online supplementary material (see www.karger.com/doi/10.1159/000505620) (Fig. 1) [9].

\section{Results}

Table 1 displays participants' characteristics. Of the 28 participants interviewed, in the randomised controlled trial, 11 were mobile teledermoscopy-enhanced SSE group participants and 17 were naked-eye SSE group participants. The interviews ranged from 5 to $10 \mathrm{~min}$. Fiftyfour percent of participants were female $(n=15 / 28)$, and their mean age was 42.2 years (range 18-60). Most participants had very fair or fair skin type $(85.7 \%, n=24 / 28)$. Prior to participating in this study $67.9 \%(n=19 / 28)$ of participants reported they had already performed SSE previously.

Five main themes were identified from the interviews: history of clinical skin examinations, continuity of the doctor-patient relationship, expedited review enhancing the doctor-to-doctor setting, convenience of the directto-consumer setting and partner-assisted task.

\section{History of Clinical Skin Examinations}

Four participants $(14.3 \%, n=4 / 28)$ had never had a clinical skin examination prior to the study (Table 2). Seven participants $(25.0 \%, n=7 / 25)$ currently had clinical skin examinations annually. The remaining participants had had $>1$ clinical skin examination previously. Previous sun exposure and having been diagnosed with a previous skin cancer were mentioned to impact frequency of clinical skin examinations. One participant commented "Well in the 5 years after my cancer, I was probably more vigilant in those 5 years ... Then after that, so when I was roughly 30 , I've had skin checks every 12 months or so" (female). A participant who had never had a clinical skin examination but had been planning to get one explained, "(it's on the) to do list, to go see the GP" (female).

Consumer Skin Cancer Screening

Preferences
Table 1. Participant characteristics

\begin{tabular}{|c|c|}
\hline Characteristic & $n(\%)$ \\
\hline Total, $n$ & 28 \\
\hline Mean age (range), years & $42.2(18-60)$ \\
\hline \multicolumn{2}{|l|}{ Study group } \\
\hline Intervention & $11(39.3)$ \\
\hline Control & $17(60.7)$ \\
\hline \multicolumn{2}{|l|}{ Gender } \\
\hline Male & $13(46.4)$ \\
\hline Female & $15(53.6)$ \\
\hline \multicolumn{2}{|l|}{ Educational attainment } \\
\hline Completed senior high school & $6(21.5)$ \\
\hline Trade or technical certificate & $7(25.0)$ \\
\hline University degree/diploma & $15(53.6)$ \\
\hline \multicolumn{2}{|l|}{ Marital status } \\
\hline Living with partner & $23(82.1)$ \\
\hline Living without partner & $5(17.8)$ \\
\hline \multicolumn{2}{|c|}{ Previous skin spot, mole or other spot removed? } \\
\hline Yes & $14(50.0)$ \\
\hline No or unsure & $14(50.0)$ \\
\hline \multicolumn{2}{|l|}{ Skin type } \\
\hline Very fair & $8(28.6)$ \\
\hline Fair & $16(57.1)$ \\
\hline Medium & $4(14.3)$ \\
\hline Dark & - \\
\hline \multicolumn{2}{|l|}{ Number of moles } \\
\hline None & $1(3.6)$ \\
\hline A few $(<20)$ & $7(25.0)$ \\
\hline Some $(20-50)$ & $11(39.3)$ \\
\hline Many (>50) & $9(32.1)$ \\
\hline \multicolumn{2}{|c|}{$\begin{array}{l}\text { Have your or someone who is not a doctor, such as a spouse or } \\
\text { partner, deliberately checked any part of your skin for early signs } \\
\text { of skin cancer? }\end{array}$} \\
\hline Yes & $19(67.9)$ \\
\hline No & $9(32.1)$ \\
\hline
\end{tabular}

Participants could select if they visit one or more types of doctors for clinical skin examinations. Fourteen participants $(50.0 \%, n=14 / 28)$ visited a GP for clinical skin examinations, 12 participants $(42.9 \%, n=12 / 28)$ visited dedicated skin cancer clinics and 6 participants $(21.4 \%$, $n=6 / 28)$ visited dermatologists. Convenience and timing were important factors impacting whether or not people were having their clinical skin examinations by the same doctor as detailed in the next 2 themes.

\section{Continuity of the Doctor-Patient Relationship}

Of the 22 participants who had had 2 or more clinical skin examinations in the past, only 5 participants $(22.7 \%$, $n=5 / 22)$ visited the same doctor each time. The remaining 17 participants did not visit the same doctor for each 
Table 2. Interview responses

\begin{tabular}{|c|c|}
\hline Characteristic & $n(\%)$ \\
\hline \multicolumn{2}{|l|}{ Total } \\
\hline \multicolumn{2}{|c|}{ Previous clinical skin examinations by a doctor $(n=28)$} \\
\hline Never & $4(14.3)$ \\
\hline Annually & $7(25.0)$ \\
\hline $1-10$ & $14(50.0)$ \\
\hline $11-25$ & $2(7.1)$ \\
\hline Many & $1(3.6)$ \\
\hline \multicolumn{2}{|c|}{$\begin{array}{l}\text { Type of doctor conducting clinical skin } \\
\text { check }(n=28)^{1}\end{array}$} \\
\hline GP & $14(50.0)$ \\
\hline Dedicated skin clinics & $12(42.9)$ \\
\hline Dermatologist & $6(21.4)$ \\
\hline \multicolumn{2}{|c|}{$\begin{array}{l}\text { If you have had more than } 1 \text { clinical skin check in the past, did } \\
\text { you visit }>1 \text { doctor? }(n=22)\end{array}$} \\
\hline Yes & $17(77.3)$ \\
\hline No & $5(22.7)$ \\
\hline \multicolumn{2}{|c|}{$\begin{array}{l}\text { Would you use mobile teledermoscopy in the direct-to-consum- } \\
\text { er setting for monitoring lesions between doctors' visits? }(n=28)\end{array}$} \\
\hline Yes & $27(96.4)$ \\
\hline No & 0 \\
\hline Unsure & $1(3.6)$ \\
\hline \multicolumn{2}{|c|}{$\begin{array}{l}\text { Would you use mobile teledermoscopy in the future to self-selec } \\
\text { lesions at home suspicious for skin cancer for remote diagnosis } \\
\text { by a teledermatologist? }(n=28)\end{array}$} \\
\hline Yes & $25(89.3)$ \\
\hline No & $1(3.6)$ \\
\hline Unsure & $2(7.1)$ \\
\hline \multicolumn{2}{|c|}{$\begin{array}{l}\text { Would you use mobile teledermoscopy in the doctor-to-doctor } \\
\text { setting for referral/triage of suspicious skin lesions? }(n=28)\end{array}$} \\
\hline Yes & $27(96.4)$ \\
\hline No & 0 \\
\hline Unsure & 0 \\
\hline Data missing & $1(3.6)$ \\
\hline
\end{tabular}

clinical skin examination. Participants found themselves visiting different doctors due to: convenience; availability of doctor at the participants' preferred days/times; long wait times at their regular doctor; participant or doctor moving locations; doctor retiring or cost (such as availability of bulk billing under the Medicare system of universal health insurance where there is no cost involved in the consultation to the patient). One participant was provided annual free clinical skin examinations at their workplace, and different doctors conducted these clinical skin examinations each year.

Participants reported visiting their regular medical practitioner but seeing "whoever is free" (female). For example, participants stated "I mainly go to the mole scan clinics, so I guess it's whoever they've got rostered to work that day" (female) and "it's the same medical centre that we go to and the same skin clinic, but it's not necessarily the same doctor that we see" (female). One participant mentioned they did not visit their doctor solely for a clinical skin examination alone; however, it "... was a normal health check-up and so she (the doctor) just did it (the clinical skin examination) as part of her routine check" (female).

Participants commented on the benefits of seeing the same doctor "because obviously 1 doctor looks at you all the time and tends to know what's going on with you" (male). Another participant stated "he's the GP that's been looking after me for 10 years or so and has all my records and knows my history ..." (male). Another participant commented "The doctors move on. Or I move. But the longer I can keep the same doctor, the better because they've got my photos on file" (male).

\section{Convenience of the Direct-to-Consumer Settings}

Twenty-seven participants $(96.4 \%, n=27 / 28)$ would like to use mobile teledermoscopy in the direct-to-consumer setting for monitoring lesions between doctors' visits, and 1 participant $(3.6 \%, n=1 / 28)$ replied they were unsure (Table 2). A participant stated "someone like myself with lots of moles, it's hard to retract which ones are the bad ones, or the ones to watch; so for the doctor to mark them for me to show my partner so she can know which ones they are for the future is a great idea" (male). For monitoring of lesions, a participant commented "in terms of working out if something has changed ... It would be easier for me to analyse the differences if I had 2 separate images to compare" (female).

Twenty-five of the participants $(89.3 \%, n=25 / 28)$ would like to use mobile teledermoscopy to self-select skin lesions suspicious for skin cancer at home and send to a teledermatologist for remote assessment, while 2 participants $(7.1 \%, n=2 / 28)$ were unsure and 1 participant $(3.6 \%, n=1 / 28)$ replied they would not like to use it (Table 2). The most cited reason for direct-to-consumer settings was convenience. A participant explained that "sometimes it might take me weeks to get in to see a GP. It might expedite that, if you've got something and you're worried about it ..." (male). Another participant commented "if there's 1 or 2 spots that comes up and you really don't want to go to the doctor to just have a look at 1 or 2 spots if you had that there that you could send through and then continue to monitor" (female). Additionally, a participant commented "(if) I have a concern I can immediately pull out the device and check it without having 
to go through the process of thinking I have to book into a doctor's appointment with a schedule" (male). This setting was appealing to many participants. "It wouldn't replace them (in-person clinical skin examinations), but yes, it would let me get some sort of care without having to go out of my way to go see a doctor" (female).

Other advantages of mobile teledermoscopy facilitating direct-to-consumer settings include that it was "a useful tool to identify changes" (male) and would "encourage me to do more skin checks" (female). It would not cause participants to worry more "I don't worry any more or any less" using the technology because "I'm an Australian, I've been in the sun forever and a day - I know I'm going to have skin damage - I just want to get it (skin cancer) soon (detected)" (female). One participant explained mobile teledermoscopy was more comfortable, “... because I'd rather do (clinical skin examinations) myself than go to a clinic and just stand there pretty much naked ..." (female). To make the process more convenient for all parties involved, several participants speculated about the potential for this technology to use automated algorithms that could provide an immediate diagnosis. One interviewee commented "if you're taking a photo of it, and it's referencing it against a database ... it might come up and say this is very similar to these other 10 samples, and yeah it's not the type you've got to worry about" (male).

\section{Expedited Review Enhancing the Doctor-to-Doctor Setting}

Twenty-seven participants $(96.4 \%, n=27 / 28)$ were agreeable to the idea that mobile teledermoscopy could be used in the doctor-to-doctor setting where GPs send photos to dermatologists to get a second opinion about skin lesions they are concerned about but not certain whether they should be referred (also called triage teledermatology). The 1 remaining participant was not asked about this setting due to the interview ending early (this was the last question). A participant commented "it could help reduce that waiting list ... to go down and see your specialist; so it's a good idea" (male). The doctor-to-doctor setting was regarded as beneficial because "you could save everyone time and money" (male). Furthermore, another interviewee stated patient wait times could be reduced "... if benign things can just be ticked off ... there's a spare appointment ... (then it is) probably easier to get (future) appointments" (female).

\section{Mobile Teledermoscopy Is a Partner-Assisted Task}

A participant commented that conducting wholebody SSEs for mobile teledermoscopy without a partner was difficult "... but it was hard to do a full skin check with yourself" (female). A partner was needed especially to photograph lesions in hard-to-see areas such as the back. One participant commented they required their partner to keep an accurate timeline, "my partner will be able to keep an accurate timeline of particular spots of interest" (male). Another participant appreciated that when a partner takes a photo of their lesion in a hard-tosee area it allows both them and the person with the lesion to view the lesion closely through the photo. “... it just gives a really good picture of it, because you can't see your back, you can't even really see it properly when you look in a mirror. To be able to take a photo is fantastic, so I really believe that's going to be the way of the future" (female).

\section{Other Patient Opinions or Comments}

In the randomised controlled trial, participants were asked to select lesions they identified as suspicious for skin cancer and send them directly to the study doctor. A participant asserted that in contrast to a health professional, lay people lacked the appropriate knowledge and education to select the correct lesions to photograph. Another participant stated that “... I wouldn't know what I was looking for” (female). Similarly, another participant preferred to use the mobile teledermoscopy device in the future for monitoring doctor-identified lesions "only if I had guidance on what I should be monitoring ... because the ones that I was monitoring - turns out none of them are really the right kind" (female). One participant commented "it could discourage me from doing full body checks. It would probably give me a bit of a false sense of security" (female). One participant commented they have less trust in the telediagnosis compared to in-person clinical skin examinations: "No ... I'm not convinced ... I just figured I'd just go to the doctor ... more trust" (male). Another participant noted they would continue with inperson clinical skin examinations due to habit "not because feels like check-up is more valuable ... but out of habit" (female).

\section{Discussion}

Mobile teledermoscopy was found to be a highly valued and attractive health technology for participants in this study. Almost all participants would be interested in integrating both the direct-to-consumer and doctor-todoctor settings into their skin cancer screening routines. Most participants had had one or more clinical skin ex- 
aminations previously and were visiting different doctors for in-person clinical skin examinations usually due to convenience. GPs were the most visited for clinical skin examinations, closely followed by skin cancer clinics run by GPs, while fewer visited dermatologists. Convenience and avoiding an in-person visit as well as having a record of skin lesions to refer back to and being able to view lesions that are otherwise invisible to the person themselves through the photographs were other cited advantages of direct-to-consumer mobile teledermoscopy. However, requiring a partner to assist with SSE for hard-to-see areas, patient education and trust in SSEs and the teledermatologist were mentioned as obstacles to mobile teledermoscopy use.

Expediting dermatologist appointments for urgent cases and reducing the wait list by removing unnecessary examinations for obviously benign cases were major arguments mentioned by the interviewed study participants for the integration of mobile teledermoscopy into the doctor-to-doctor setting. Other benefits to this setting were raised including: expediting doctor visits earlier if the telediagnosis advised the lesion required treatment, ease of access to a specialist doctor, time savings avoiding an in-person visit and ability to monitor suspicious lesions more frequently. In a previous study the melanoma referral pathway was expedited using a virtual lesion clinic (9 days compared to 26.5 days for standard outpatient assessment) [10]. A recent review reported teledermatology programmes that supported image-forwarding to dermatologists are effective as a triaging tool, reducing face-to-face referrals by 31-88\% [3]. A large-scale telemedicine network in Brazil found almost half of the patients who were reviewed through teledermatology could be treated locally by their primary care physicians [11]. In this model, the doctor-patient relationship is preserved as the primary care provider has access to the teledermatology results and will treat the patient as per the dermatologist instructions, while providing continuity of care. The benefit of teledermoscopy can be further seen by patients living in geographically disadvantaged areas [3]. However, teledermoscopy is still foreign to some GPs, and the additional workload, technical and reimbursement issues may pose a challenge to many GPs practising this service [12].

Participants expressed they would enlist the help of their partners to help monitor their skin lesions, especially to assist with photographing hard-to-see areas. This also implies they may reciprocate by monitoring their partners' lesions as well, thus promoting healthy habits. Barriers to mobile teledermoscopy that participants expe- rienced in this study included patient education to recognise suspicious skin lesions and trust issues in the teledermoscopy process; however, these issues were only mentioned by a small number of participants $(11 \%, n=3 / 28)$ because a question about trust was not in the interview guide.

Eber et al. [13] reported that only $20 \%$ of dermatologists in Austria were willing to perform teledermatology for an initial consultation and that barriers of teledermatology included the lack of personal contact $(64.2 \%, n=$ $156 / 243)$, legal uncertainties $(54.7 \%, n=133 / 243)$ and the lack of reimbursement $(45.7 \%, n=111 / 243)$. In a previous Australian study assessing doctors' perspectives of direct-to-consumer patient-initiated teledermoscopy, doctors were critical or unsure $(77.3 \%, n=34 / 44)$ whether it would provide a good service [12]. Consumers could attend an in-person appointment with their doctor to commence the process and receive educational training. If the doctor is confident the consumer can monitor their lesions confidently, then future visits can be reduced but not replaced completely. Janda et al. [12] previously reported doctors would prefer patients to use it for monitoring doctor-identified lesions, rather than detection of a new lesion.

A potentially detrimental consequence often cited with regard to telemedicine is the impact on the doctorpatient relationship [14]. Positive relationships between doctors and patients are important because they have been shown to be associated with better patient outcomes and satisfaction [15]. The continuity of the traditional doctor-patient relationship is evolving in an increasingly time-pressured society in combination with the influence of telemedicine or mHealth apps [16]. In primary care, patients value a patient-centred approach, which $\mathrm{mHealth}$ apps aim to provide, most importantly assisting with effective communication, partnership and health promotion [17]. mHealth apps rather than depersonalising health care could potentially foster doctor-patient relationships by helping to increase continuity and frequency of contact with providers. It can be argued that online communication may lack the familiarity and comfort a patient requires in a therapeutic relationship with their doctor. However, a systematic review reported after patients adopted mHealth apps they felt empowered and informed and felt it had a positive impact on the relationship with their medical providers [16].

\section{Strengths and Limitations}

This study gained a deeper understanding of participants' skin cancer screening behaviours and their atti- 
tudes towards the use of different modes of use for mobile teledermoscopy services. Data saturation was achieved in a large sample of 28 participants, and the analysis was carried out by 2 researchers independently. However, this study has some limitations. The interviews were part of a larger study about mobile teledermoscopy-assisted SSE. Participants were aware they would possibly receive mobile teledermoscopy services and were therefore motivated to use the technology, otherwise they would not have participated in the study. Participants who elected to join the study received a whole-body skin examination and may therefore be more motivated to engage with clinical skin examinations compared to the general population.

\section{Conclusion}

Participants gave favourable comments for direct-toconsumer and doctor-to-doctor mobile teledermoscopy settings. In this study, the conventional personal doctorpatient relationship was not a leading priority for clinical skin examinations with many participants visiting several medical practitioners. Convenience, access and cost were factors which contributed to the casual approach to clinician continuity for clinical skin examinations in this study. In the direct-to-consumer setting, mobile teledermoscopy may complement the SSE process through monitoring of doctor-identified lesions to reduce in-person visits. Ultimately, the implication of teledermoscopy on the doctor-patient relationship is still uncertain. It may potentially be detrimental through reduced face-toface consultations, but the relationship may be improved through more convenient communication and improved feedback from dermatologists.

\section{Key Message}

Mobile teledermoscopy was viewed positively by patients for both direct-to-consumer and doctor-to-doctor interactions.

\section{Acknowledgements}

This project was undertaken as part of an NHMRC partnership research project on behalf of Queensland University of Technology (QUT) and the University of Queensland (UQ), in partnership with FotoFinder Systems GmbH, the Princess Alexandra Hospital Foundation, Melanoma Patients Australia, Queensland Institute of Dermatology, Skin and Cancer Foundations Inc. and Skin and Cancer Foundation NSW and the Dermatology Departments of the University of Graz, University of Arizona and Memorial Sloan Kettering Cancer Center, USA.

\section{Statement of Ethics}

This study was approved by the Queensland University of Technology Human Ethics Committee (QUT approval No. 1400000807).

\section{Disclosure Statement}

H.P.S. is a shareholder of MoleMap NZ Limited and e-derm consult $\mathrm{GmbH}$, and undertakes regular teledermatological reporting for both companies. H.P.S. is a Medical Consultant for Canfield Scientific Inc. and MetaOptima Technology Inc., and a Medical Advisor for First Derm.

\section{Funding Sources}

The study is funded by a research grant awarded to M.J. from the National Health and Medical Research Council (NHMRC) APP1113962. M.J. is funded by an NHMRC TRIP Fellowship APP1151021. H.P.S. has an MRFF Next Generation Clinical Researchers Program Practitioner Fellowship APP1137127.

\section{Author Contributions}

M.J., C.H. and H.P.S contributed to trial conception and design. M.J. and J.R. designed the interview guide. M.S. conducted the interviews. Thematic analysis was conducted by C.H. and F.K.; C.H, M.O. and M.J. drafted the manuscript. All authors provided critical revision of the manuscript for important intellectual content.

\section{References}

1 Tensen E, van der Heijden JP, Jaspers MW, Witkamp L. Two Decades of Teledermatology: Current Status and Integration in National Healthcare Systems. Curr Dermatol Rep. 2016;5(2):96-104.

2 Koh U, Horsham C, Soyer HP, Loescher LJ, Gillespie N, Vagenas D, et al. Consumer Acceptance and Expectations of a Mobile Health Application to Photograph Skin Lesions for Early Detection of Melanoma. Dermatology. 2019;235(1):4-10.
3 Lee KJ, Finnane A, Soyer HP. Recent trends in teledermatology and teledermoscopy. Dermatol Pract Concept. 2018 Jul;8(3):214-23.

4 Ngoo A, Finnane A, McMeniman E, Soyer HP, Janda M. Fighting Melanoma with Smartphones: A Snapshot of Where We are a Decade after App Stores Opened Their Doors. Int J Med Inform. 2018 Oct;118:99-112. 
5 Chuchu N, Dinnes J, Takwoingi Y, Matin RN, Bayliss SE, Davenport C, et al.; Cochrane Skin Cancer Diagnostic Test Accuracy Group. Teledermatology for diagnosing skin cancer in adults. Cochrane Database Syst Rev. 2018 Dec;12:CD013193.

6 Manahan MN, Soyer HP, Loescher LJ, Horsham C, Vagenas D, Whiteman DC, et al. A pilot trial of mobile, patient-performed teledermoscopy. Br J Dermatol. 2015 Apr;172(4): 1072-80.

7 Dobson J. Co-production helps ensure that new technology succeeds. BMJ. 2019; 366:14833.

8 Chao JT 2nd, Loescher LJ, Soyer HP, CurielLewandrowski C. Barriers to mobile teledermoscopy in primary care. J Am Acad Dermatol. 2013 Nov;69(5):821-4.

9 Janda M, Horsham C, Koh U, Gillespie N, Loescher LJ, Vagenas D, et al. Redesigning Skin Cancer Early Detection and Care Using a New
Mobile Health Application: Protocol of the SKIN Research Project, a Randomised Controlled Trial. Dermatology. 2019;235(1):11-8.

10 Congalton AT, Oakley AM, Rademaker M, Bramley D, Martin RC. Successful melanoma triage by a virtual lesion clinic (teledermatoscopy). J Eur Acad Dermatol Venereol. 2015 Dec;29(12):2423-8.

11 von Wangenheim A, Nunes DH. Creating a Web Infrastructure for the Support of Clinical Protocols and Clinical Management: An Example in Teledermatology. Telemed J E Health. 2019 Sep;25(9):781-90.

12 Janda M, Horsham C, Koh U, Gillespie N, Vagenas D, Loescher LJ, et al. Evaluating healthcare practitioners' views on store-and-forward teledermoscopy services for the diagnosis of skin cancer. Digit Health. 2019 Feb;5: 2055207619828225.

13 Eber EL, Janda M, Arzberger E, HofmannWellenhof R. Survey on the status of teleder- matology in Austria. J Dtsch Dermatol Ges. 2019 Jan;17(1):25-31.

14 Finnane A, Dallest K, Janda M, Soyer HP. Teledermatology for the Diagnosis and Management of Skin Cancer: A Systematic Review. JAMA Dermatol. 2017 Mar;153(3):31927.

15 Saultz JW, Lochner J. Interpersonal continuity of care and care outcomes: a critical review. Ann Fam Med. 2005 Mar-Apr;3(2): 159-66.

16 Qudah B, Luetsch K. The influence of mobile health applications on patient - healthcare provider relationships: A systematic, narrative review. Patient Educ Couns. 2019 Jun; 102(6):1080-9.

17 Little P, Everitt H, Williamson I, Warner G, Moore M, Gould C, et al. Preferences of patients for patient centred approach to consultation in primary care: observational study. BMJ. 2001 Feb;322(7284):468-72. 